Journal of Universal Computer Science, vol. 18, no. 1 (2012), 5-24

submitted: 15/7/11, accepted: 9/12/11, appeared: 1/1/12 @ J.UCS

\title{
Exploiting Semantics for Constructing and Comparing Occupational and Educational-driven Qualifications: the TIPTOE Project
}

\author{
Valentina Gatteschi, Fabrizio Lamberti, Claudio Demartini \\ (Politecnico di Torino, Dip. di Automatica e Informatica, Torino, Italy \\ valentina.gatteschi@polito.it, fabrizio.lamberti@polito.it \\ claudio.demartini@polito.it) \\ Rob van Wezel \\ (KCH International, Horapark 2, 6717 LZ EDE, The Netherlands \\ r.vanwezel@kch.nl)
}

Simonetta Bettiol

(Ufficio Scolastico Regionale per il Veneto, Riva de Biasio S. Croce 1299, 30135

Venezia, Italy

simonetta.bettiol@istruzioneveneto.it)

\begin{abstract}
In recent years, mobility of students and workers started to be considered as a way for answering skill shortages in the European labor world. However, in order to implement effective mobility practices, suitable instruments supporting transparency and readability of the outcomes of learning processes as well as of the needs of companies and employers in general have to be developed. In fact, though some instruments have been introduced (like, for instance, the European Qualification Framework, EQF, the European Credit system for Vocational Education and Training, ECVET, etc.), they are often seen as theoretical tools rather than a practical help for involved stakeholders. In this work, the results of the TIPTOE project, a transnational initiative funded by the European Commission under the Lifelong Learning Programme are illustrated. In particular, the semantic-based methodology for the construction of a European-wide profile mixing education and labor worlds perspectives in the trade sector is discussed. Furthermore, a number of tools allowing end-users to compare owned qualifications to the reference one and supporting stakeholders in the reading of national educational and occupational profiles in the EQF dimension are presented. Key Words: Taxonomy, ontology, lifelong learning, qualifications, semantic engine Category: I.2.1, I.2.4
\end{abstract}

\section{Introduction}

During last years, issues related to mobility of students and workers across Europe gained more and more relevance. Nowadays people have better chances to spend a studying or working period abroad to acquire missing competences, or to find better working opportunities. However, marked differences in the meaning, content and interpretation of tasks and functions as well as of learning outcomes 
in the framework of the European labor market and educational offer still limit the mobility of workers and learners. In this context, the correct identification of competences fulfilling training gaps, as well as the depiction of acquired competences (according to a shared formalism) becomes of primary importance for an individual.

For this reason, since the establishment of the European Union, several instruments have been developed in order to guarantee comparability, transferability and recognition of qualifications across different countries, as well as to enhance transparency and mutual understanding across Member States. Efforts in this field tackled various school levels, from compulsory education and initial vocational education and training to adult and higher education, etc.

One of the most relevant tools in this context is the European Qualification Framework (EQF) [EQF, 2008], a common reference system conceived to support the linking of different countries' national qualifications systems and frameworks together. According to the EQF, lifelong learning qualifications are categorized in eight reference levels, and associated learning outcomes are described in terms of knowledge, skills and competence concepts, thus opening the way for the creation of a shared understanding in the lifelong learning domain.

Thanks to the EQF, qualifications (as well as curricula, syllabi, etc.) described according to the above guidelines have more possibilities of being understood and referenced by all the actors involved in the educational and occupational domains. Nevertheless, when it is needed to compare education and training contents or personal abilities for various mobility purposes, a mere application of the EQF principles (e.g., carried out on a manual basis) may not be feasible, since a huge amount of information has to be considered. This is the case, for instance, of job placement or recognition of prior learning scenarios, where personal abilities owned by a given applicant have to be analytically compared to individual components of a reference profile. Hence, ad hoc instruments, able to semantically compare information embedded into qualifications, course profiles, résumés, etc. by exploiting the EQF guidelines should be created.

The goal of this paper is to present the approach pursued within the TIPTOE "Testing and Implementing EQF and ECVET Principles in Trade Organizations and Education" project (01.10.2008-30.11.2010) ${ }^{1}$ to address the above need. The project was coordinated by Kenniscentrum Handel, a centre of expertise for vocational education and training in the Netherlands that works in collaboration with companies and educational institutions to develop and maintain the national qualification structure for existing and new occupations in the trade sector. The project involved training centers of France, United Kingdom, the Netherlands, Italy, Lithuania, Portugal, Germany, Belgium and Slovenia as well national social partners and authorities. Politecnico di Torino was responsible

\footnotetext{
${ }^{1}$ NL/08/LLP-LdV/ TOI/123011, http://www.evta.net/tiptoe/home_tiptoe/
} 
for the developments based on the use of semantic technology.

In a nutshell, the project aimed at lowering existing barriers between the labor market and the training dimension at the European level, by identifying a common European profile in a specific scenario (in the particular case represented by the trade sector) to be exploited for experimenting EQF-based automatic processing of relevant information in the mobility perspective. As it will be detailed in the following, the approach moved from the construction of a semantic engine able to perform an EQF-aware ontological comparison of country-based formative offers (expressed through educational profiles) and labor market requirements (represented by national occupational profiles) with the goal of finding similarities and specificities emerging from heterogeneous "local" descriptions structured in terms of learning outcomes. Through the engine, the common profile was created, and specific web-based services allowing users (that could be either education and training Authorities and institutions, companies, job-seekers as well as students) to compare their own qualifications and profiles to the European profile were developed.

The rest of the paper is organized as follows. Section 2 provides an overview of research activities exploiting semantic paradigms to support education and recruitment/job-seeking activities. Section 3 summarizes the objectives of the TIPTOE project, whereas Section 4 illustrates the four-step methodology developed for the creation of the common profile. Finally, Section 5 discusses some of the main services offered by the project platform, whereas Section 6 draws conclusions and presents future research directions and concrete exploitation steps that are currently in progress.

\section{Related works}

As briefly presented previously, the objective of this work is to illustrate a methodology to be used for identifying common elements or differences between European occupational and training worlds, by exploiting core elements belonging to the semantic web field. In particular, with the aim of supporting the comparison and linking of qualifications, core concepts such as ontological descriptions (defined by Gruber as explicit specifications of a conceptualization [Gruber, 1993]) have been exploited. Moreover, in order to define relations among concepts and, thus, to improve the comparison and linking processes, a taxonomy (a classification arranged in a hierarchical structure) has been created.

The application of semantic paradigms to working and learning domains has already been investigated. [Poyry et al. 2002, Poyry and Puustjarvi, 2003] presented a first example showing how metadata could be exploited to support learners looking for European higher education courses that match their needs. A different solution, that goes beyond the strategy in the works mentioned above 
is depicted in [Nemirovskij et al. 2007]. In this work, a semantic search strategy based on the analysis of the relations among concepts belonging to user queries and concepts used in learning documents is explained. A different approach aimed at supporting students' mobility is presented in [Hoffman, 2008]. Here, the author suggests an interesting use of taxonomies for comparing European engineering courses. In particular, an adaptation of the Bloom's learning taxonomy [Bloom, 1956] for organizing verbs is investigated, and a representation of skills as pairs of noun-verb elements is presented. A further approach showing a general-purpose strategy for measuring the differences among qualifications by defining meta-ontologies describing referencing rules between national models is presented in [Gatteschi et al. 2009]. In this work, formal models of national education and training systems are created, and meta-ontologies are exploited for overcoming the heterogeneity of qualification structures belonging to different countries. In [Chen et al. 2008], another interesting methodology aimed at reducing the cognitive overload disorientation that may arise by the inappropriate curriculum sequencing during personalized Web-based learning is presented. In particular, a novel genetic-based curriculum sequencing scheme based on a generated ontology-based concept map is discussed. Another interesting strategy, exploited to manage and maintain Medicine curricula, is reported in [Dexter and Davies, 2009]. In this work, the authors present an ontology-based knowledge base able to deal with the multi-dimensional matrix depicting relations between curriculum contents and intended learning outcomes. The issue of curriculum development has been tackled also in [Tang, 2009], where the authors define an ontology of electrical engineering curricula that takes into account correlated topics that might exist in various courses, and allows the system to identify, when a new course is added, which are the pre-requisites and the foundational courses to be added to the study plan. Another work dealing with the need for a personalized curriculum in the European perspective is reported in [Ferreira and Filipe, 2009]. Here, the authors present a common conceptual model supported by the Academic Ontology Bologna Process with the aim of allowing interoperability between academic management systems and automation of academic management. [Ronchetti and Sant, 2007] present how ontologies could be used for managing, inspecting and monitoring a full study course, by allowing a system to verify overlaps between courses, in order to find out areas which are not covered and to analyze possible synergies with courses offered in other schools. A different methodology, developed in order to enable students to integrate classes from other institutions into their curriculum, in the Bologna Process view [Bologna Declaration, 1999], is shown in [Hackelbusch, 2006]. In this work, the author presented a system, based on a curricula mapping ontology, providing students with a ranked list of classes offered by other academic institutes, by including only classes that were identified as interchangeable, from 
the organizational and the semantic point of view. Finally, [Mirizzi et al. 2009] propose the use of a domain ontology for automatically producing a semantic annotated electronic résumé. In this work, the authors show how the recruitment phases could be possibly supported by an ontology of terms, that could be used to suggest, starting from an initial set of competences specified by the user, additional competences that may be also included in the résumé.

As in some of the above works, the methodology discussed in this paper strongly relies on taxonomies and ontologies for representing qualifications and for developing semantic-based comparison strategies. However, while the objective of the above works consists generally in ranking elements according to their degree of similarity with a target description, the present work aims at identifying common elements among a huge variety of descriptions. Moreover, the current work proposes a general-purpose methodology strongly exploiting subsumption relations within a strictly structured context represented by the EQF framework and by its associated principles, and aims at investigating whether the use of classifications of concepts could improve the results of the comparison.

\section{The TIPTOE project}

Mobility of individuals can represent a powerful instrument to address existing skill shortages and mismatches in a country or region, thus improving the efficiency of the labor market and removing brakes on the economic growth. However, even though all the parties involved could benefit from transnational mobility, there are still several barriers to this process, mostly due to the differences in the meaning, content and interpretation of tasks, functions and learning outcomes to be carried out by and expected from European workers and students.

For this reason, the objective of the TIPTOE project was to tackle the problem of interpretation and application of the EQF principles from a practical perspective, by specifically focusing on a sector that is considered of primary importance for the markets and for the partnership (that is the trade one) and by proposing a methodology capable of mitigating both the gap between the different European education and training systems as well as the (cultural) differences in the content and interpretation of occupations within the European labor market.

The basic assumption behind the TIPTOE project is that labor market and educational field have both their own understanding of which kind of knowledge, skills and competences are related to a given professional. Nonetheless, frequently, qualifications provided by education and training institutions do not really reflect labor market's needs. Moreover, the lack for rules outlining a minimum set of knowledge, skills and competences that a student should possess at 
the end of a training path creates strong information asymmetries between the education and the labor worlds and severely limits mobility among countries.

In fact, since a unique and well-defined qualification profile is missing, employers may ignore the exact contents of the courses attended by a student who is applying for a given job position, and consequently, may not know which learning outcomes have been actually achieved. The depicted scenario is even more jeopardized and complex in a transnational perspective, especially when non-formal and informal learning paths are also taken into account.

Within the TIPTOE project, both the labor market and the training field are investigated and compared at the European level, with the aim of identifying the core elements (knowledge, skills and competence) characterizing a professional, which are then exploited to build the European-wide profile. The strategy pursued throughout the project strongly relies on semantic instruments for allowing an automatic and syntax-independent comparison of the above domains.

The main result of TIPTOE is the development of a knowledge base hosting the common profile and related information exploited in the construction steps. Moreover, a number of services targeted to involved actors have been developed for supporting the construction of the profile and enabling for its possible extension (details will be provided in Section 4). Finally, based on such a knowledge base, a number of services targeted to end-users have been implemented, which allowed to validate the appropriateness of the profile and which represent today the practical interface of the project with the education and labor worlds (details will be provided Section 5). All the above functionalities can be accessed online via the project web platform ${ }^{2}$.

For what it concerns the creation of the common European profile, the methodology adopted throughout the project is the following. First, several interviews with relevant stakeholders of the labor world were carried out. The objective of this first phase was to outline a set of tasks a worker should be able to accomplish by characterizing them in terms of knowledge, skills and competences. Secondly, an investigation on the education and training field was performed. In this phase, several interviews with relevant training organisms were conducted in order to identify which learning outcomes a student should possess at the end of a formal training path. After this phase, the two sets of information had to be compared in order to identify the common elements between the requirements of the occupational domain and the outputs of the educational routes. This comparison was aimed at defining a unique profile, drawn according to the EQF principles. Then, in order to catalogue the outcomes of the interviews in a structured way and to perform the required semantic reasoning onto them (thus avoiding possibly incorrect results provided by a manual comparison carried out on a huge amount of data), elements belonging to occupational and

\footnotetext{
${ }^{2}$ http://www $\cdot$ tiptoe.polito.it/
} 
educational descriptions have been linked to a set of concepts, organized into a taxonomy. The resulting ontology, which shows the links among elements of the descriptions and concepts of the taxonomy, allowed to carry out the reasoning by exploiting the relations among elements and, thus, overcoming lexical barriers.

Based on the outcomes of the above phases, several services specifically addressed to project stakeholders were developed. In particular, in order to support the users in the identification of the EQF level for a given qualification, a specific tool for the automatic referencing has been implemented. Moreover, results provided by the semantic engine have been collected and structured according to their EQF level in a EQF ruler, so that to provide a quick and easy-to-read representation (navigation) of the common profile.

\section{The methodology}

The methodology devised for the creation of the common European profile consists of four stages, namely 1) information collection, 2) taxonomy and ontology construction, 3) definition of inference rules and approaches for semantic comparison and, finally, 4) common profile creation. In the following, each stage will be discussed in details by making reference to Figure 1, where the overall methodology is summarized.

\subsection{Information collection}

The information collection stage was carried out in order to collect the requirements of the labor world and the outputs of the education and training domain, expressed in terms of units, tasks, subtasks as well as of knowledge, skill and competence elements.

In order to define a shared format for collecting information (and then representing it in the taxonomy and ontology construction stage) the representation of knowledge, skill and competence concepts made by [Pernici et al. 2006] was exploited. According to [Pernici et al. 2006], a knowledge could be defined as a set of knowledge objects (KO), a skill could be represented as a KO "put into action" through an action verb (AV), hence by one or more pairs $\mathrm{KO}-\mathrm{AV}$, and a competence could be identified as a triple $\mathrm{KO}-\mathrm{AV}-\mathrm{CX}$, which describes the ability of putting into action a given KO in a specific context (CX). The relations among the above concepts have been taken into account during the information collection phase.

In particular, four relevant professional profiles were identified in the selected sector (namely Shop Assistant, Shop Manager, Logistic Assistant and Logistic Manager), and several interviews were conducted in both the working and the training contexts in order to identify their key elements. At a first stage, stakeholders (i.e., employers of the retail and wholesale sectors) belonging to the 

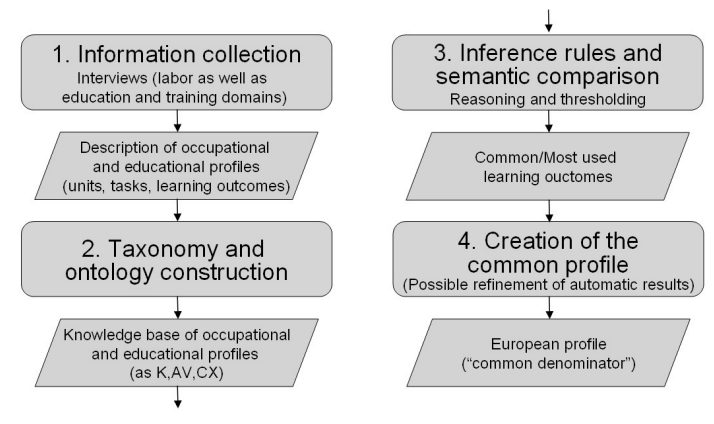

Figure 1: Overall methodology for the creation of the common profile

labor dimension of different European countries (i.e., France, United Kingdom, the Netherlands, Italy, Lithuania, Portugal, Germany, Belgium and Slovenia) were interviewed in order to collect, for each of the four profiles, a list of knowledge, skills and competences that a worker must possess for fulfilling a task, each characterized by the corresponding EQF level (depicting the complexity degree). Subsequently, the education domain was investigated by interviewing education and training Authorities of the eight countries above, with the aim of collecting information regarding learning outcomes achieved by the students at the end of a specific training route.

Results of this phase have been collected and inserted in several grids, that can be accessed from the section "Research results per country" on the project web platform ${ }^{3}$. An example is reported in Figure 2.

\subsection{Taxonomy and ontology construction}

In this phase, collected profiles have been manually inspected in order to identify core elements (knowledge objects, action verbs and context elements) to be used for constructing the ontology. In particular, each instance of knowledge, skill and competence elements has been expressed as a combination of one or more concepts (or keywords).

Then, identified concepts were linked to each other by subsumption relations in a taxonomic representation composed of three families of terms hierarchically structured (trees), i.e., knowledge objects, action verbs and context.

For the creation of the knowledge and context trees, it was necessary to start from scratch, since the existing taxonomies were not able to fully satisfy our requirements. On the contrary, for the representation of the action verbs tree, an adaptation of the Bloom's taxonomy composed of six families of verbs (arrange, act, prepare, check, assess and react) was exploited.

\footnotetext{
${ }^{3}$ http://www.evta.net/tiptoe/home_tiptoe/research.htm
} 


\begin{tabular}{|c|c|c|c|c|c|c|c|}
\hline Part C & & $\begin{array}{c}\text { Knowledge } \\
\text { theoretical and/or factual knowlec }\end{array}$ & & $\begin{array}{c}\text { Skills } \\
\text { cognitive and practical skills }\end{array}$ & & $\begin{array}{c}\text { Competence } \\
\text { responsibility and autonomy }\end{array}$ & \\
\hline $\begin{array}{l}\text { Subtask } 4: 3.4 \text {. Checking } \\
\text { best before dates and the } \\
\text { condition of merchandise; } \\
\text { stock rotation }\end{array}$ & 2,5 & $\begin{array}{l}\text { Excellent knowledge of } \\
\text { hygiene and (if applicable) } \\
\text { fresh products handling and } \\
\text { storing procedures. } \\
\text { Knowledge of company } \\
\text { procedure of best before } \\
\text { dates, quality standards for } \\
\text { the condition of merchandise } \\
\text { and stock rotation (in case of } \\
\text { fresh stock: newer in the } \\
\text { back, older in front, etc). }\end{array}$ & 2,5 & $\begin{array}{l}\text { For products where } \\
\text { esthetical presentation is } \\
\text { important, a sense of colour } \\
\text { and style in order to set up } \\
\text { the shelves in an attractive } \\
\text { way, but conveying an } \\
\text { impression of new } \\
\text { merchandise. }\end{array}$ & 2 & $\begin{array}{l}\text { Precision, especially in the } \\
\text { case of fresh merchandise. } \\
\text { All fresh stock should be } \\
\text { taken off the shelves } 6 \text { days } \\
\text { prior to the best before date. } \\
\text { Older stock should be in front } \\
\text { on the shelves, newer stock } \\
\text { in the back. }\end{array}$ & 3 \\
\hline
\end{tabular}

Figure 2: Grid created for the Shop Assistant profile from Italy (excerpt)

It is worth remarking that in the definition of the taxonomy, experts from the trade sector have been highly involved, since an improper hierarchy of keywords could lead to incorrect results in the next phases.

Then, after the creation of the taxonomy, qualifications, tasks and subtasks were described by linking their composing elements (knowledge, skills and competences) to the corresponding concepts (knowledge objects, action verbs and context elements).

For this purpose, in order to provide a formal and easy-to-read description to be shared with the involved actors and stakeholders, a graphical representation of the ontology was drawn by exploiting the UML notation and the open-source tool $\mathrm{UMLGraph}^{4}$, a software that is able to process diagrams expressed in a textual form and to draw the corresponding graphical representation. UML diagrams were embedded in the platform so as to allow users to browse work or education related maps during the population of the knowledge base.

Figure 3 shows an excerpt of the subtask To welcome the customer and understand the customer's needs and requests, belonging to the Shop Assistant profile from Portugal. In particular, the diagram displays the knowledge Communication techniques knowledge, the two skills To be able to apply selling techniques and To be able to communicate in English, and the competence Full responsibility in identifying the customer and his needs. In order to better characterize knowledge, skill and competence elements, the corresponding classes are shown in dark gray, while the concepts of the taxonomy they are linked to are painted light gray. In addition, subsumption relations are expressed by a solid line with a hollow arrowhead pointing from the class that is subsumed to the class that subsumes. Finally, the fact that a knowledge, skill or competence is characterized by one or more concepts from the taxonomy is denoted by a dashed line.

The diagram should be read as follows. The knowledge Communication tech-

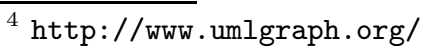




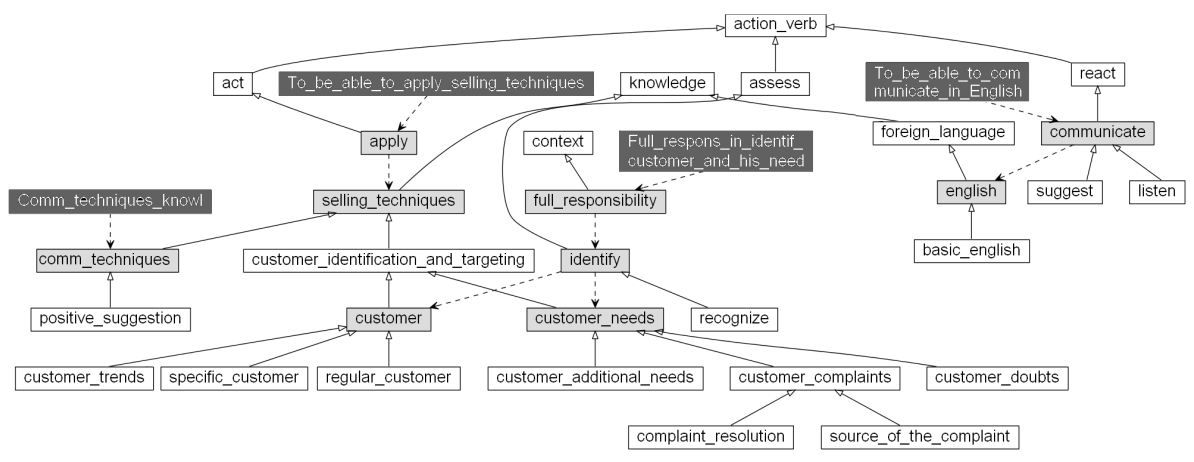

Figure 3: Portion of the ontology related to the subtask To welcome the customer and understand the customer's needs and requests

niques knowledge is linked to the knowledge object communication techniques that is a type of selling techniques, i.e., another knowledge object. The subsumption relation between selling techniques and communication techniques shows that if, for instance, a student has got a communication techniques knowledge, then the student also knows something about selling techniques. Furthermore, the skill To be able to apply selling techniques is characterized by the pair of concepts apply, an action verb that further specifies the action verb act, and selling techniques, a knowledge object, whereas the skill To be able to communicate in English is defined by the action verb communicate, a specification of the action verb react, which is applied to the English concept, a specification of a generic foreign language knowledge object. Finally, the competence Full responsibility in identifying the customer and his needs is described by a full responsibility context, applied to the identify action verb, which is linked to customer and customer needs knowledge objects.

\subsection{Inference rules and approaches for semantic comparison}

The basic idea behind the definition of the inference rules required for the TIPTOE project is the following. Since the common profile must act as a "common denominator" among different profiles, it should be a combination of elements that are somehow expressed in all the profiles and, as a consequence, it should be the sum of all the knowledge, skills and competences that are linked to the most used knowledge objects, action verbs or context elements.

An example could probably explain in a clearer way the above statement. Let us consider four subtasks belonging to four profiles, defined by the knowledge elements cleaning techniques knowledge, cleaning means and tools knowledge, cleaning methods knowledge and cleaning methods, means and tools. Since each 
profile contains (at least) a knowledge that is related to the cleaning activity, this knowledge should also be included in the common profile. On the contrary, if a particular knowledge (e.g., the product lifecycle knowledge) is mentioned in just one profile, it shall not be incorporated in the common profile.

In addition, the reasoning that has been presented above should be based on the keywords linked to the elements belonging to the profile descriptions. In fact, a semantic engine should be able to understand that the four knowledge elements mentioned above are linked to the cleaning concept (then, in the ontology, they will be characterized by the cleaning knowledge element).

It is clear that the common profile will then be a representation of the most common knowledge, skill and competence elements. Consequently, the engine for semantic comparison should be able to identify the most used keywords, recognize which elements they are linked to, and then include these elements into the common profile. A further step towards the achievement of a more correct result could be the exploitation of the taxonomy of terms and subsumption relations. In this way, by analyzing the example shown in Figure 1, the number of occurrences of communication techniques, customer, customer needs, English, apply, identify, communicate and full responsibility would be 1, while the number of occurrences of the (parent) element selling techniques would be 4 , since the selling techniques concept has been exploited once, but the (children) elements communication techniques, customer and customer needs have been used each one once too.

Four comparison strategies have been developed and investigated in order to find the best result. All of them take as input a threshold (that is a minimum number of times a keyword is used) defined by the user, and explore the ontology in order to identify the most common elements. The four comparison strategies developed are simple range, simple range with mean, aggregate simple range and aggregate range with mean.

The simplest way of determining which knowledge, skill and competence elements will belong to the common profile is the simple range strategy, since it calculates a value that corresponds to the number of times a keyword has been linked to the elements of the ontology. If this value is higher than the threshold defined by the user, the strategy includes the considered knowledge, skill or competence into the common profile.

A slightly more complex approach is the simple range with mean. According to this strategy, the value computed by the comparison tool (which, in order to add the element belonging to the common profile, must be higher than the threshold defined by the user) is the average of the number of occurrences of each keyword linked to the knowledge, skill or competence being considered.

A third approach, which takes into account also hierarchical relations expressed by the taxonomy, is the aggregate simple range. According to this strat- 


\begin{tabular}{|l|l|}
\hline Strategy & Results \\
\hline Simple range & 38 \\
\hline Simple range with mean & $13.5=(38+12+3+1) / 4$ \\
\hline Aggregate range & $122=(38+84)+(12+14)+3+1$ \\
\hline Aggregate range with mean & $38=[(38+84)+(12+14)+3+1] / 4$ \\
\hline
\end{tabular}

Table 1: Results obtained from the application of the four comparison strategies to the Knowledge of products and relevant display techniques (i.e., volume displays and on shelf couponing) element with a threshold equal to 10

egy, the tool calculates the number of times a keyword, and the subsumed concepts, have been used to describe the elements of the ontology. If this value is higher than the threshold specified by the user, the examined element is added to the common profile.

A fourth strategy, that is similar to the simple range with mean and that allows to consider also subsumption, is the aggregate range with mean. According to this approach, the value computed by the comparison tool is the mean of the number of occurrences of each keyword and its children in the taxonomy.

A further example could help to understand the logic behind the four different approaches. Let us consider the Knowledge of products and relevant display techniques (i.e., volume displays and on shelf couponing) element and let us assume that this knowledge is described by the keywords product (used 38 times in the profile descriptions), exposition techniques (used 12 times), volume displays (used 3 times) and on shelf couponing (used only in this description). Furthermore, let us suppose that the product and the exposition elements have several children in the knowledge taxonomy, and that the respectively subsumed concepts have been used 84 times and 14 times, respectively.

If the simple range strategy is adopted, the result would be 38 , that is the maximum value of occurrences of the keywords linked to the knowledge element. On the other hand, the result of the simple range with mean approach would be 13.5, that is the average of the occurrences of the four keywords linked to the element. When subsumption relations are considered, the computed value would increase. In fact, the result of the aggregate range approach would be 122, that is the sum of the occurrences of product (122, that is $38+84)$, exposition (26, that is $12+14$ ), volume displays (3) and on shelf couponing (1) concepts. Finally, if the strategy applied is the aggregate range with mean, the result would be 38, that is the average of the values above.

It is worth remarking that the results just discussed (and shown in Table 1) represent only an estimate of how common a knowledge, skill or competence is. For the particular profiles collected in the knowledge base, the most accurate 
results were produced by the aggregate range with mean, as learning outcomes were often expressed by making reference to action verbs quite common (like apply) and words definitely less common (given the specificity of the domain). Nonetheless, a given value could not be good or worst a priori, since it would have to be compared with the other results. Consequently, possible ways for the identification of the common profile could be to order the results from the one that obtained the highest value to the one that got the lowest one, and then select a number of elements defined by the user (i.e., the number of knowledge, skill and competence elements in the common profile would be fixed), or - and this was the case for the project - to use the threshold expressed by the user to select only those elements that achieved a score higher than it.

\subsection{Creation of the common profile}

The common profile was created running the semantic engine and exploiting the reasoning approach explained in the previous stage. According to the above discussion, the knowledge, skill and competence elements obtaining a specific value became potential components of the common profile. It is worth observing that this step is carried out in a completely automated way. However, since - like in the case of the above example with the set of knowledge elements described by the cleaning techniques concept - it would have been redundant inserting into the common profile four elements with the same meaning, we decided to let the user eventually choose, among the set of elements exploiting the same keywords, the one that could better represent the specific knowledge, skill or competence.

Figure 4 shows an example of knowledge described by the knowledge object cleaning techniques. Here, the user selected as the representative knowledge the element cleaning methods. As shown in Figure 5, statistics on the exploitation of single or aggregate keywords (for a single country, as well as for the whole working or training dimensions) may be exploited in this step. Also, UML maps can be navigated to get insights on specific portions of national maps regarding the education and labor domain as well as their relations with the overall profile being created (as already shown in Figure 3).

For the whole profile ${ }^{5}$, an EQF level is computed as an average of the EQF values assigned to each knowledge, skill or competence selected as potential component of the whole profile.

\section{Services for end-users and validation of the methodology}

In addition to the features described in the previous section, a tool for the automatic identification of the EQF level of a new qualification based on information

${ }_{5}^{5}$ http://www.tiptoe.polito.it/wholecommonprofile.php 


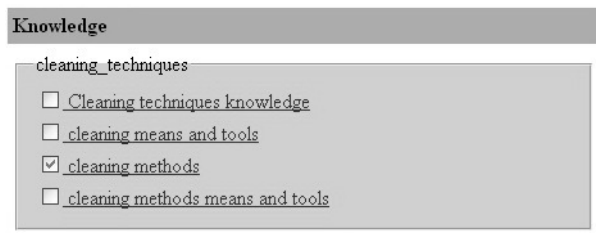

Figure 4: Selection of the elements that will belong to the common profile

stored in the knowledge base has been implemented. This tool has also been used to perform a content-based validation of the overall methodology and to evaluate the performance of the semantic-based reasoning. Furthermore, the EQF ruler, a navigable collection of shared learning outcomes for the trade sector structured according to their EQF level, has been developed. Details of the above services, which are mainly targeted to end-users, are discussed in the following.

\subsection{Automatic identification of the EQF level}

The tool for the automatic identification of the EQF level allows users to insert in the platform a new profile, and to exploit the semantic engine (used for the construction of the common profile) for the automatic identification of the corresponding EQF level. In this way, an immediate comparison of the owned knowledge, skills and competences with respect to the European reference can be obtained. The underlying idea behind the development of this instrument is that, for each learning outcome belonging to a given profile, an EQF level could be automatically identified by considering the EQF level previously assigned to similar learning outcomes.

For testing the tool, the referencing of an international trade profile developed by the TIPTOE coordinator in the framework of the COMINTER "Creation and implementation of a common European qualification in international trade" project ${ }^{6}$ following a methodology derived from the Sustainable Professionalisation $^{7}$ experience was selected as a test bed. First, the core elements (units, tasks, subtasks and learning outcomes) belonging to the COMINTER profile were inserted into the platform.

Afterwards, starting from the information collected in the knowledge base, the suite for the identification of the EQF level has been created. The recognition of the correct level is carried out by performing a semantic search on concepts expressing the meaning of the unlabeled learning outcome, in order to identify whether the knowledge base yet contains some knowledge, skills or competence

\footnotetext{
6 http://www . cominter-europe.org/

7 http://www.trainingvillage.gr/etv/Projects_networks/CCprojects/dipl_ methodofinale_FR.pdf
} 


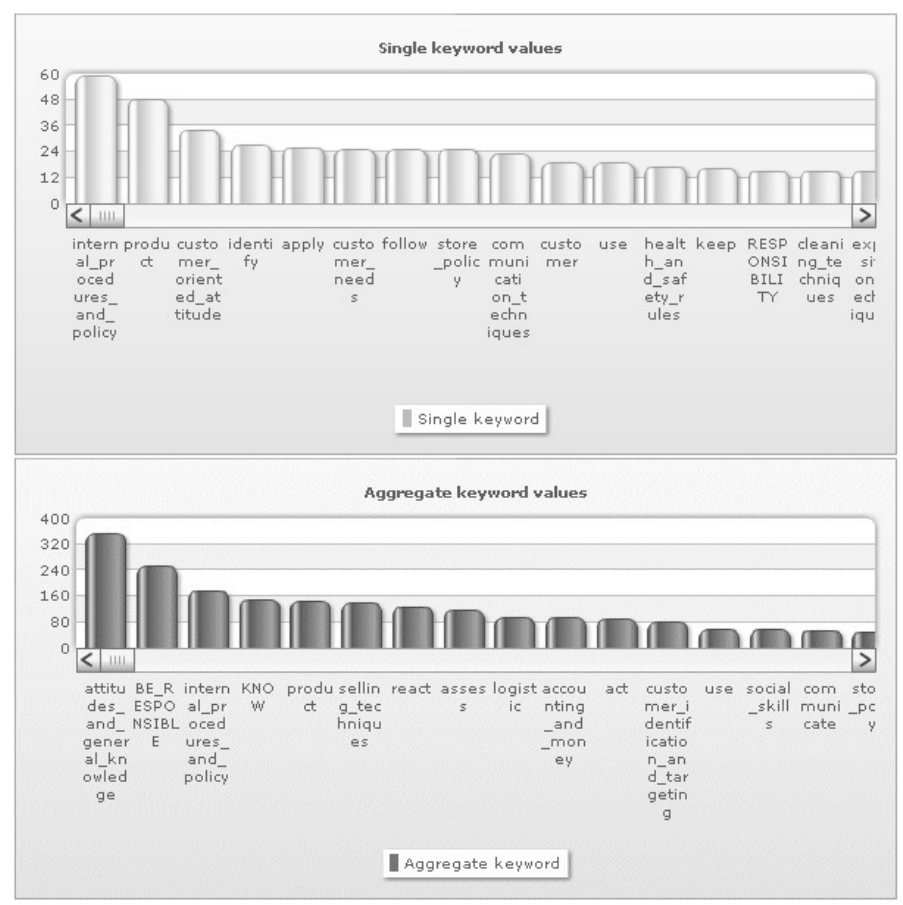

Figure 5: Statistics on the exploitation of concepts in the description of learning outcomes characterizing the Shop Assistant job profile

(with an EQF level previously specified) exploiting the same keywords used for the description of the COMINTER element. However, since a perfect match between the searched learning outcome and the already inserted knowledge, skill and competence elements is nearly rare (because both elements should have been described by the exact set of concepts), it is evident that a search engine should also browse the taxonomy (in order to identify more specific and more general concepts). Moreover, scenarios in which only a part of the whole set of terms is contained in the semantic description of a learning outcome must be considered.

An example could better clarify the above statement. Let us consider a skill To be able to recognize the needs of a customer whose EQF level is unknown and let us imagine that the linked keywords expressing its meaning are recognize and customer needs. The search engine should look inside the knowledge base in order to identify all the elements described by that exact set of terms. Even though these concepts are frequently used, let us suppose that no other learning outcomes are described by the couple recognize - customer needs, but there are some skills expressing the ability of identifying customer needs (thus, described by the couple identify - customer needs), and that the underlying taxonomy 
of terms somehow defined a relation between the recognize (more specific) and the identify (more general) concepts, as already depicted in Figure 3. In this case the former skill is a specification of the latter skill, since, according to the taxonomy, the activity of recognizing customer needs implicitly requires the ability to identify customer needs. Hence, the two skills could be considered as similar, and the EQF value of the latter could be directly assigned to the former.

In order to achieve the expected result, the tool for the identification of the EQF level first identifies all the possible combinations among the sets of concepts used for the description of a given learning outcome (i.e., KO, AV and CX) by also browsing the taxonomy. Then, it performs a search in the knowledge base to identify the presence of already inserted knowledge, skills and competences described by one (or more) combinations of terms (in this example, possible combinations are recognize - customer needs, recognize - customer identification, recognize - selling techniques, identify - customer needs, identify - customer identification, identify - selling techniques). It is worth remarking that, in this phase, different knowledge, skills or competences already present in the knowledge base could be identified, according to the different combinations of terms (for example, several learning outcomes containing the couples recognize - customer needs or identify - customer needs could be found). In this case the tool privileges the ones described by the more specific concepts (i.e., recognize - customer needs), since they are closer to the meaning of the unlabeled element.

Once the reference learning outcomes have been picked out, the EQF level is computed as a mean among the values characterizing them. However, together with this value, additional information concerning its reliability should be displayed to the user. Hence, a measure of the distance between the searched concepts and the concepts found in the taxonomy is reported. This value gives an idea of the remoteness of two elements and it is minimal in the case of coincident concepts. Thus, for two couples of concepts recognize - customer needs and identify - customer needs the distance will be equal to one, since, in the taxonomy, the identify AV is only one level higher than the recognize AV.

Another possible scenario the user should be aware of occurs when only a limited number of concepts describing an element of the new profile is contained in the description of a knowledge, skill or competence. Let us clarify the above statement with the additional example of an unlabeled skill to be able to recognize the needs and the expectations of a customer. In this case the meaning of the learning outcome is expressed by the set recognize customer needs and customer expectations. Let us compare this skill with an already inserted one, like to be able to identify customer needs, and let us imagine that this is the closest element in the knowledge base (hence, no other learning outcomes described by the three concepts or by a combination of their higher level elements could be identified). In this case, even though skills denote similar things, it is evident that the first is 


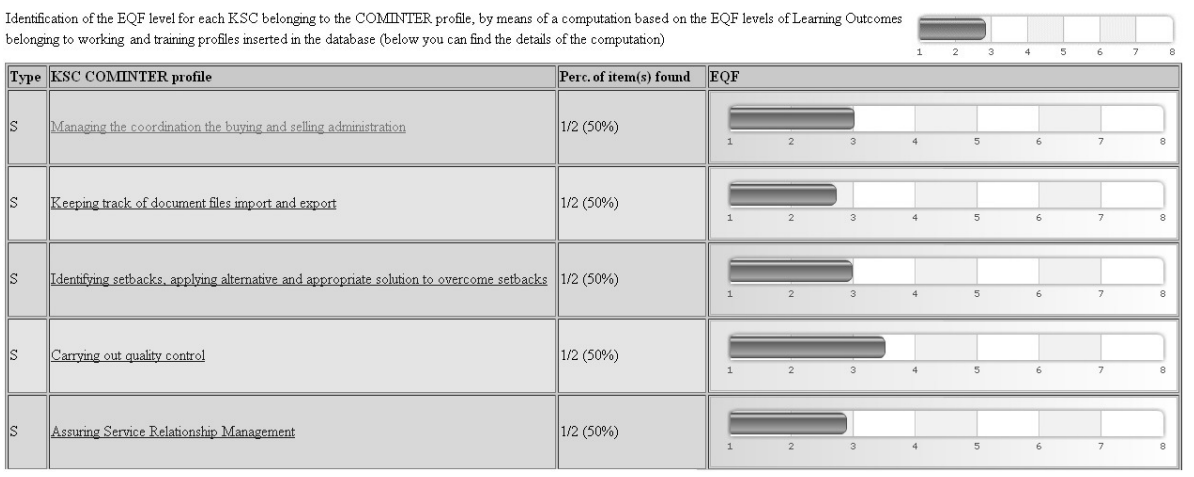

Figure 6: Results provided by the tool for the identification of the EQF level

more complex than the second one, since it requires also the ability to recognize customer expectations. This peculiarity should be communicated to the user, as an indication of the accuracy of the EQF level that has been identified. Hence, together with the result, a value reporting the percentage of concepts found in the semantic description of a learning outcome is also shown.

Figure 6 shows a snapshot of the page computing the EQF level for different aggregations (learning outcomes, units, etc.). As a matter of example, the EQF value assigned to the considered unit is slightly less than 3 , since it is the average of the levels of the composing knowledge, skills and competences.

Besides allowing to validate the tool for the automatic assignment of the EQF level, the experiments carried out with the COMINTER profile additionally provided an implicit verification of the effectiveness of the overall TIPTOE methodology. In fact, the EQF level of the COMINTER profile had already been determined by the experts before introducing it in the knowledge base and the tool, by exploiting the designed reasoning, automatically assigned the same level.

It is worth remarking that, while, on the one hand, end-users could directly benefit from such an instrument, on the other hand, each time they insert a new profile for determining its EQF level, they contribute to broaden the knowledge base itself. Hence, a massive exploitation of the TIPTOE web portal simultaneously contributes at enriching contents and improving results.

\subsection{The EQF ruler}

The EQF ruler is a shared collection of learning outcomes for the trade sector structured according to their EQF level and to the type of the task they are referred to, which has been developed in order to provide stakeholders with an easy way to access the core outcome of the project, i.e., the European profile. 


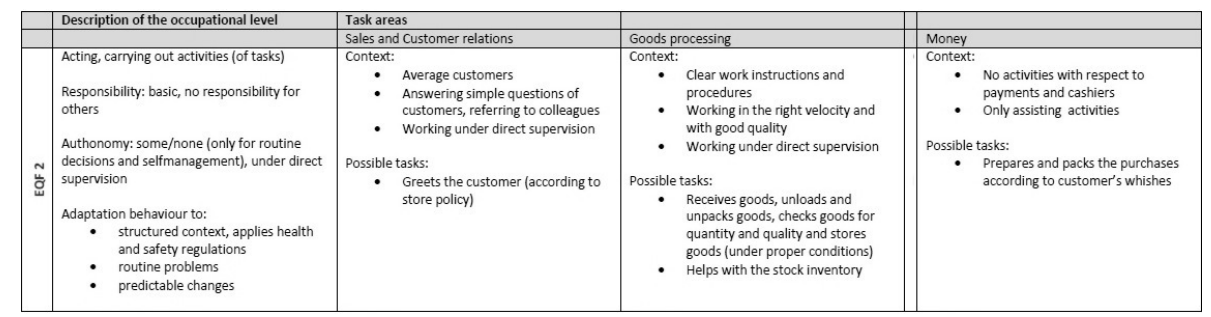

Figure 7: EQF ruler: occupations for the retail dimension (excerpt)

For what it concerns occupations related to the retail dimension, five task areas have been identified, namely sales and customer relations, goods processing, presentation, promotion and marketing, money and shop management. Figure 7 shows an excerpt of the EQF ruler created for the Shop Assistant and Shop Manager occupations. The first column displays a synthesis of the required degree of autonomy and responsibility, whereas the remaining columns provide information on the context and possible tasks linked to each task area (only EQF level 2 learning outcomes and a subset of task areas are shown).

The EQF ruler additionally proved to be a good way to help stakeholders familiarize with the EQF. In fact, the framework is often seen as a theoretical instrument, and involved parties find it extremely difficult to exploit it practically (e.g., for recruitment, job-seeking, etc.). A survey on project stakeholders confirmed that this instrument was fully appreciated as a tool that simplifies the whole referencing process since it tells the user the work context of an individual per level. Hence, it could be considered as a valid starting point for professional users as it allows them to discuss EQF levels issues by making them more visible and easily perceived.

\section{Conclusions and future works}

In the present work, a semantic-based methodology for the construction of a common EQF-aware European profile in the trade sector merging contributions from national educational and occupational profiles has been presented. In particular, the knowledge base hosting the common profile together with the main services provided by the web platform developed within the project have been discussed. The objective of the devised system is twofold. On the one hand it supports involved actors in the creation of a common profile for qualifications related to the European trade sector. On the other hand, it provides end-users with a set of services supporting them in the analysis of qualifications expressed according to the EQF principles. For what it concerns the creation of the common European profile, the four steps of the pursued methodology are explained, 
whereas, regarding the services tailored to end-users, an instrument for the automatic identification of the EQF level, together with the EQF ruler have been presented. The common profile created within the project could lower barriers linked to information asymmetries between European labor and training dimensions, as it defines a common basis for the construction of qualifications belonging to the European trade sector. Moreover, the suite of services for the automatic identification of the EQF level could support end-users in the analysis of a new profile, and could better assure transparency during qualification recognition phases. Additionally, the EQF ruler could be used by them during interviews with job candidates in order to assign an EQF level to learning outcomes characterizing job applicants' grounding, thus making them fully understand whether the person in front of them is too much or not enough skilled, with respect to a searched job profile.

Future works will be devoted to an extension of the proposed approach to other contexts. In particular, in the framework of TAMTAM "Exploiting the TIPTOE plAtforM by transferring ECVET and EQF semAntic tools in a Multi-sectoral perspective" project ${ }^{8}$ started on October 1st, 2011 and involving various former TIPTOE partners, the methodology discussed in this work will be transferred in both the geographic and sectoral perspectives. In particular, the TIPTOE approach will be applied to the ICT, fashion/textile, mechanics/mechatronics and energetic efficiency/green building areas by considering national profiles from countries not involved in TIPTOE, like Austria and Spain.

\section{Acknowledgements}

Contents reported in this work refer to the activities carried out in the context of the TIPTOE "Testing and Implementing EQF and ECVET Principles in Trade Organizations and Education" project. The work has been prepared as a dissemination activity of the TAMTAM "Exploiting the TIPTOE plAtforM by transferring ECVET and EQF semAntic tools in a Multi-sectoral perspective" project (2011-1-IT1-LEO05-01969 - CUP G12F11000600006) funded with the support from the European Commission under the Lifelong Learning Programme - Leonardo da Vinci - Transfer of Innovation 2011 call. This work reflects the views only of the authors, and the Commission cannot be held responsible for any use which may be made of the information contained therein.

\section{References}

[Bloom, 1956] Bloom, B.S.: "Taxonomy of educational objectives - the classification of educational goals - Handbook I: cognitive domain"; Longman, (1956)

\footnotetext{
8 http://www.evta.net/tiptoe/home_tiptoe/research.htm
} 
[Bologna Declaration, 1999] "The Bologna Declaration (19 June 1999)"

[Chen et al. 2008] Chen C.M., Peng C.J., Shiue J.Y.: "Ontology-based Concept Map for Planning Personalized Learning Path"; IEEE Conference on Cybernetics and Intelligent Systems (ICCIS 2008), 1337-1342 (2008)

[Dexter and Davies, 2009] Dexter, H., Davies, I.: "An ontology-based curriculum knowledgebase for managing complexity and change"; 9th IEEE International Conference on Advanced Learning Technologies, 136-140 (2009)

[EQF, 2008] "Recommendation of the European Parliament and of the Council of 23 April 2008 on the establishment of the European Qualifications Framework for lifelong learning"

[Ferreira and Filipe, 2009] Ferreira, J.C. and Filipe, P.: "Academic Ontology to Support the Bologna Mobility Process"; 2nd International Conference on Adaptive Science \& Technology (ICAST 2009), 308-313 (2009)

[Gatteschi et al. 2009] Gatteschi, V., Lamberti, F., Salassa, F., Demartini, C.: "An Automatic Tool Supporting Life-long Learning Based on a Semantic-Oriented Approach for Comparing Qualifications"; IADIS International Conference on Cognition and Exploratory Learning in Digital Age (CELDA 2009), 354-358 (2009)

[Gruber, 1993] Gruber, T.R.: "Toward principles for the design of ontologies used for knowledge sharing"; Padua workshop on Formal Ontology, 1-23 (1993)

[Hackelbusch, 2006] Hackelbusch, R.: "Handling Heterogeneous Academic Curricula: National qualification systems integration using ontologies"; 17th Int. Workshop on Database and Expert Systems Applications (DEXA 2006), 344-348 (2006)

[Hoffman, 2008] Hoffmann, M.: "Using Bloom's Taxonomy of learning to make engineering courses comparable"; EAEEIE 19th Annual Conf. (2008), 205-209 (2008)

[Mirizzi et al. 2009] Mirizzi, R., Di Noia, T., Di Sciascio, T.: "A Semantic Web enabled System for Résumé Composition and Publication"; IEEE International Conference on Semantic Computing (ICSC 2009), 583-588 (2009)

[Nemirovskij et al. 2007] Nemirovskij, G., Egner, M.T., Heuel, E.: "SWAPS: Semantic Web Approach for Personalisation of Study"; 7th IEEE International Conference on Advanced Learning Technologies (ICALT 2007), 711-712 (2007)

[Pernici et al. 2006] Pernici, B., Locatelli, P., Marinoni, C.: "The eCCO System: An eCompetence Management Tool Based on Semantic Networks"; Workshop on Ontology Content and Evaluation in Enterprise (OnToContent 2006), 1088-1099 (2006)

[Poyry et al. 2002] Poyry, P., Pelto-Aho, K., Puustjarvi, J.: "The role of metadata in the CUBER system"; Proc. of the 2002 annual research Conf. of the South African institute of computer scientists and information technologists on Enablement through technology, 16-18 (2002)

[Poyry and Puustjarvi, 2003] Poyry, P., Puustjarvi, J.: "CUBER: a personalised curriculum builder"; Proceedings of the 3rd IEEE Int. Conf. on Advanced Learning Technologies, 326-327 (2003)

[Ronchetti and Sant, 2007] Ronchetti, M., Sant, J.: "Curriculum Management and Review: an ontology-based solution"; World Conference on E-Learning in Corporate, Government, Healthcare, and Higher Education (ELEARN 2007), 6476-6482 (2007)

[Tang, 2009] Tang, A.: "An Ontological Approach to Curriculum Development"; International Conference on Engineering Education (ICEED 2009), 219-224 (2009) 